\title{
Linéaments d'écriture. Les ratures dans la correspondance de peu-lettrés durant la Grande Guerre
}

\author{
Stéphanie Fonvielle*, \\ CNE-UMR 8562, Aix-Marseille Université \\ 2, rue de la Charité - 13236 Marseille cedex 02 \\ France
}

\begin{abstract}
Résumé. Cet article se propose d'analyser les ratures dans des correspondances peu-lettrées de la Grande Guerre. L'objectif est de voir comment ces dernières peuvent refléter les conditions de production des lettres et cartes échangées entre les soldats et leur famille. Nous présentons dans un premier temps la singularité du contexte générique dans lequel apparaissent ces ratures, précisons leur mode de repérage au moyen d'un logiciel textométrique et explorons, à l'aide des critères proposés par la génétique des écrits, la valeur indicielle de certaines ratures. Une seconde partie sera l'occasion pour nous d'entrer plus avant dans les bifurcations des écrits de peu-lettrés, en interrogeant notamment les degrés de contrainte et de liberté qui guident l'instance raturante, en évoquant la part des ratures liées à l'orthographe, et enfin, en sillonnant quelques réécritures proposées par les épistoliers peu-lettrés, qui nous conduisent du côté du lapsus.
\end{abstract}

\begin{abstract}
Over several writing lineaments. The erasures in lowliteracy correspondences of the Great War. This article proposes to analyze the erasures in low-literacy correspondences of the Great War. The objective is to see how these can reflect the conditions of production of letters and cards exchanged between soldiers and their families. We first present the singularity of the generic context in which these erasures appear, specify their mode of identification using textometric software and explore, using the criteria proposed by the genetics of the written word, the index value of certain erasures. A second part will be the occasion for us to go further into the bifurcations of the writings of low-literate people, by questioning in particular the degrees of constraint and freedom which guide the erasing instance, by evoking the part of erasures linked to spelling, and finally, by going through some rewritings proposed by lowliterate letter writers, which lead us to the side of the slip.
\end{abstract}

\footnotetext{
*Stephanie.fonvielle@univ-amu.fr
} 


\section{Introduction}

« ceula ne se ra pas trop tot quand tous çela finira ch que nous pourron terminer toute les écriture lointaine, comme ça on poura se causer de vive voix » (Laurent Pouchet, 14 avril 1915). Dans sa lettre, ce poilu exprime sa lassitude à communiquer par écrit. L'exercice pour lui est d'autant plus éprouvant qu'il ne possède, pour exprimer son quotidien, que les rudiments d'une écriture apprise à l'école primaire de la troisième République. Dans ses lettres, comme dans celles des autres poilus peu-lettrés (Branca-Rosoff et Schneider, 1994), l'on reconnait d'une lettre à l'autre les mêmes formules liminaires, vestige du canon générique scolaire (Vicari, 2018 ; Große et al, 2016). Les poilus ont beaucoup écrit durant la Grande Guerre, on le sait. Depuis les tranchées ou l'hôpital, en déplacement ou en cantonnement, ils pouvaient rédiger plusieurs lettres et cartes par jour, quelques mots pour montrer qu'ils sont en vie, ou de longues missives pour raconter leur guerre ordinaire. Par endroits, une rature fissure leur propos, elle « est un effacement visible, un silence audible, et donc, une trace visible» (Grésillon, 2000: 49). Ces ratures que nous nous proposons d'étudier ici, sont en effet bien plus qu'un trait tracé sur du dit, elles représentent des points de bifurcation dans l'écrit des peu-lettrés, matérialisent l'" avoir-eu-lieu » de leur geste scriptural (Rougé, $2000: 7$ ). Nous proposons de voir comment quelques ratures glanées dans des lettres échangées entre des familles de peu-lettrés séparées par la Grande Guerre peuvent renseigner sur la gestuelle et la pratique scripturaire de ces épistoliers occasionnels.

\section{Variations sur la rature}

Notre analyse des ratures épistolaires s'inscrit dans un cadre exploratoire qui croise trois paramètres. Paramètre situationnel d'abord, constitué par le contexte génétique singulier dans lequel apparaissent les ratures, celui des écrits peu-lettrés durant la Grande Guerre. Paramètre formel ensuite, qui interroge la traçabilité des ratures dans ces lettres et cartes, le mode de repérage d'une opération de mise sous silence de l'écrit, ou de son absence. Un paramètre indiciel enfin, qui, notamment grâce aux outils développés par la génétique et la linguistique de l'écrit, investit la rature d'une valeur signifiante.

\subsection{Les correspondances de peu-lettrés dans la Grande Guerre}

Les ratures à l'étude proviennent d'un corpus de lettres et cartes postales échangées durant la Première Guerre mondiale, entre des soldats qui sont au front ou à l'hôpital et leur famille restée à l'arrière ${ }^{1}$. Ce fonds épistolaire comprend actuellement ${ }^{2} 1797$ lettres ou cartes échangées entre vingt-six épistoliers ${ }^{3}$ originaires de l'Hérault, de l'Ain, de l'Ardèche, des Charente-Maritime, de la Marne et de Saône et Loire. Des correspondances entre les membres d'une même famille ont été retrouvées. Parmi elles, celle de Marie Fabre, une paysanne solarienne qui écrit à son mari Pierre, hospitalisé à la suite d'une blessure au bras droit, et à son frère Ernest Viste, lequel rassure depuis les tranchées sa femme Marguerite et sa fille Elise ; ou encore celle de Laurent Pouchet, un viticulteur héraultais, qui envoie ses tendres baisers à ses parents, sa belle-sœur, et son épouse Joséphine. La fiche matricule des soldats situe leur degré d'instruction au niveau 2 ou 3 de la classification de l'armée ; ces soldats mobilisés savent «lire, écrire» ou «lire, écrire, compter». Cultivateurs, charron, agriculteurs ou viticulteurs, l'écriture ne faisait pas partie d'une pratique quotidienne, et leurs écrits présentent les caractéristiques d'un «parlé graphique» (Steuckardt, 2014) $)^{4}$. Ces quelques lignes consacrées à la description du «corpus 14 » précisent la singularité du contexte génétique dans lequel apparaissent les ratures à l'étude. 
Ces dernières, relevées au sein de lettres qui relèvent d'une écriture ordinaire ${ }^{5}$ (Fabre, 1993), peuvent révéler les conditions dans lesquelles les scripteurs écrivent au quotidien. Ces conditions de production, nous les connaissons pour partie, grâce aux témoignages des épistoliers qui décrivent leur situation d'énonciation, s'excusant parfois au passage de ne pas pouvoir faire plus long ou de faire court. Ils disent notamment l'importance de l'instant épistolaire, souvent un «petit moment ${ }^{6}$ niché dans les interstices de leur quotidienneté : Ernest Viste écrit pendant que le café se prépare, dans le baraquement de tranchée ; Marie Fabre, en gardant les vaches ou « au pied du feu » le temps que le souper cuise ; à l'hôpital, Pierre profite d'un moment entre deux tours de manille. D'autres contraintes sont évoquées, qui circonscrivent un peu plus le contexte génétique des ratures observées, des contraintes physiques, comme un vaccin ou une blessure qui ankylose le bras, ou des contraintes matérielles. Les soldats ou leur famille évoquent ainsi à l'occasion la rareté du papier, qui peut les amener à suspendre momentanément l'échange épistolaire : «un mot encore avant de te quitter car j'ai plus de papier » (Alfred Foray, 20 octobre 1914) ; « laursque tu écrira a la maison tu leur demandera cil non pas du papier pour écrire car de puis le 27 jai reçu juste une lettre » (Alfred Foray, 10 octobre 2015). La rareté du médium invite à reconsidérer la nature même des lettres du corpus relativement au modèle du genre, qui entretient un rapport modeste avec l'usage de la rature, traditionnellement réservée au brouillon ${ }^{7}$. Pas de mention de brouillon pour la plupart de nos scripteurs peu-lettrés ${ }^{8}$, dont on peut supposer qu'ils écrivent leurs lettres et cartes sans filet.

\subsection{La traçabilité de la rature}

Pour évaluer le nombre de ratures présentes dans les lettres du «Corpus 14 », nous avons utilisé le logiciel de textométrie TXM. Ce dernier intègre différentes versions du «Corpus 14 », où figurent retranscrites toutes les cartes et lettres des scripteurs peu-lettrés. Peuvent y être consultées aussi leurs fiches d'identification, comprenant leur prénom, nom, date et lieu de naissance, leur date et lieu de décès, le numéro de matricule des soldats, leur profession et leur situation familiale. L'approche textométrique permet une analyse qualitative (collocation de mots $^{9}$ ) ou quantitative (fréquence de lemmes ${ }^{10}$ ) des textes encodés. Nous avons pour notre part extrait par requête l'ensemble des marques de reprises graphiques balisées dans la version « corpus $1412 \mathrm{~T}$ », qui rassemble 1241 lettres et cartes de 26 épistoliers, et comptabilise 324178 mots (ponctuation comprise). Au moment où nous avons effectué cette recherche, le logiciel faisait état de 784 reprises, parmi lesquelles 757 ratures, qui prennent la forme de biffures horizontales et 27 surcharges, par lesquelles l'épistolier remplace un segment par un autre segment en réécrivant par-dessus. Les reprises concernent moins de $5 \%$ des mots du corpus. Ce pourcentage, s'il indique dans l'absolu une fréquence basse, n'en reste pas moins signifiant au regard du contexte génétique du corpus (supra 2.1). Sur ces lettres destinées à être lues parfois à voix haute figurent donc des traces de reprise, qui renvoient à deux gestes différents du scripteur : soit ce dernier trace un trait définitif sur le terme à corriger, soit, beaucoup plus rarement, il réécrit par-dessus. Une ventilation par scripteur du nombre et du type de reprises effectuées distingue, suivant leur degré de reprises, les scripteurs dont la reprise fait partie du processus d'écriture, de ceux qui y ont peu, voire pas, recours. Joseph Ramier et Alphonse $^{11}$ font ainsi partie des épistoliers qui raturent le plus (+ d'1\% de leurs mots), tandis que Ernest Viste, Alfred et Emile Foray ou Victorin Folier reprennent très peu leurs écrits $(0,02 \% \text { pour le premier, } 0,07 \% \text { pour les quatre suivants })^{12}$. Les lettres de cinq épistoliers ${ }^{13}$ ne présentent quant à elles aucune marque de reprise. Le logiciel ne nous a pas permis, en l'état, d'affiner certaines recherches concernant les reprises, et a contraint certains de nos résultats. Le ratio des ratures a été calculé relativement au mot, unité comptabilisée par le logiciel. Or, l'étendue des ratures varie dans le corpus, certaines 
concernent une lettre, d'autres le mot entier. Impossible de distinguer les ratures selon leur étendue donc. L'absence même de ratures dans certains contextes où elles seraient a priori attendues, dans le cas des doublons par exemple, demeure impossible à tracer dans la version actuelle de TXM. Un relevé manuel doublé d'une analyse qualitative s'est imposé alors, pour définir dans quelle mesure et de quelle manière les ratures des épistoliers «(ir)radient ${ }^{14}$.

\subsection{La valeur indicielle de quelques ratures}

Les ratures ne sont pas que tracé, elle font trace au sens où elles relèvent d'un paradigme de l'indice (Ginzburg, 1980). Nous souhaitons ici révéler la portée signifiante de quelques ratures en les passant au crible génétique. Nous empruntons à la génétique des textes quelques critères d'analyse, qui réfèrent à l'identité de la rature, à son étendue, sa temporalité, à l'opération scripturale qu'elle signale ${ }^{15}$. Les ratures à l'étude présentent des caractéristiques communes. Elles sont quasiment toutes autographes ${ }^{16}$, figurent sur des supports manuscrits, des lettres et des cartes postales ${ }^{17}$, et s'inscrivent dans le contexte génétique de la correspondance privée de la Grande Guerre. Autre point commun, les ratures et les surcharges laissent le segment biffé vi-lisible, pas de ratures à visée occultante, ou caviardage, dans les lettres. Au niveau de leur fonction, les ratures renvoient à des gestes fondamentaux du processus d'écriture : l'épistolier rature pour éliminer définitivement un segment écrit (rature de suppression en (1)) ou pour supprimer un segment écrit puis le remplacer par un autre segment écrit ${ }^{18}$ (rature de substitution en (2)). Un troisième type de rature, la rature anticipatoire (infra 3.3.) consiste à raturer temporairement un mot, qui sera rétabli à l'identique plus loin dans la phrase (3) :

(1) il ne vale rien du je ne peut pas les mettre (Laurent Pouchet, 14 novembre 1914)

(2) je crois bientôt que la misére va venir dans <a> la campagne (Jules Ramier, 10 mai 1915) ${ }^{19}$

(3) Je te qu dit que un un ces jours je te R'onvoy erai de l'argent (Jules Ramier, 17 janvier 1914) ${ }^{20}$ Le corpus comprend des ratures d'étendue restreinte, elles ne dépassent pas le plus souvent le périmètre du $\operatorname{mot}^{21}$. La rature peut cibler une lettre ou un graphème (4), une partie du mot (5) ou le mot entier (6) :

(4) tu à l'łaire de me le dire sale mal rasé et maigre (Alfred Foray, 19 octobre 1914); tu m'en envoyies plus (Jules Ramier, 3 novembre 1916); tu tait fais fere dé $\mathrm{ph}<\mathrm{ph}>$ otot (Joséphine Pouchet, 10 mars 1915); nous somme dans les tranchées a trente mètre des allements a laur il faut $\mathrm{au}<\mathrm{ou}>$ vrir l'oeil et le bon (Laurent Pouchet, 21 novembre 1914)

(5) nous somme en trantet<ché> en ce momend (Jules Ramier, 29 juillet 1916); celui qui aura le bhon $<$ bonh $>$ eur de sans sauver il poura aller se reposer chez lui car la France en a mare et ta $<$ L'a $>$ llemagne de même (Jules Ramier, 5 juin 1916)

(6) marie a et tes quontente ta voir de tes nouvelles aucie $<$ in cie $>$. que Me Regual $<$ Rigal $>$ (Joséphine Pouchet, 21 janvier 1915)

On voit à certaines ratures le scripteur suspendre son geste d'écriture, pour effectuer une correction orthographique $(4,5,6,8$; infra 3.2.) et des rectifications d'anticipation $(3,9$; infra 3.3) :

(8) tu me dit que je pe ta voier tu vin mais non card $<$ sac $>$ oude tro cher (Joséphine Pouchet, 9 janvier 1915);

(9) peu être que dans quelle que jour sa marcheras un peu mieux (Jules Ramier, 15 novembre 1917)

Bien entendu, le support peut aussi contraindre l'écriture des épistoliers qui s'interrompent et raturent arrivés en fin de ligne $(10)^{22}$ :

(10) ne vous inqui // inquiétait pas de moi (Laurent Pouchet, 29 novembre 1914) ; par maigar // maigarde (Laurent Pouchet, 16 février 1915)

Les écrits portent enfin la marque de corrections immédiates: le substitut suit immédiatement, sur la même ligne, le terme raturé. Ces corrections portées « au fil de la 
plume, [...] [et qui] font partie intégrante du processus d'écriture par lequel le texte se crée vers l'avant» (Grésillon, Lebrave, 2003 : 137), révèlent un geste inscrit dans l'instant (supra 2.1.), une écriture que l'on devine "prise dans le mouvement du dire » (BrancaRosoff, 2015 : 51). Dans le corpus, nous n'avons pas relevé de ratures de relecture, situées hors ligne, qui seraient " postérieures au moment de l'écriture » (Grésillon, Lebrave, 2003 : $137)^{23}$. Ces ratures laissent imaginer le geste d'un épistolier engagé dans son à-écrire, pris dans « l'énergie dialectique de l'écriture » (Biasi, $2000: 19)$.

\section{Bifurcations}

Cette partie est l'occasion pour nous de voir comment les ratures signalent des choix plus ou moins conscients des scripteurs en lien avec leur pratique scripturale en temps de guerre. Les ratures trahissent la présence d'une instance raturante qui invite à interpréter les bifurcations du texte comme des nœuds de tension, des effets de normes, ou l'expression d'une contrainte.

\subsection{D'une tension l'autre}

La rature matérialise le lieu d'une tension scripturale, à différents niveaux. La rature signale tout d'abord un changement de démarche de la part du scripteur, lequel interrompt momentanément son activité proprement linguistique de mise en mots de son discours, pour passer à une activité d'ordre métalinguistique, celle de raturer : « Le fait de barrer entre dans l'opposition métalinguistique "ajouter une séquence/retrancher une séquence", les deux opérations échappant à la spontanéité de l'encodage phrastique » (Rey-Debove, 1982 :111). Analysée ensuite relativement à la dynamique de l'écriture, la portée rétroactive de la rature entraine une tension du geste scriptural, pris entre la progression d'un à écrire et la régression que suppose la reprise d'un déjà écrit. La rature est ainsi l'indice d'un changement d'orientation gestuelle du scripteur qui trace un trait sur une forme écrite ; une lettre, une syllabe ou un mot dont la forme lui semble suffisamment irrégulière ou anormale pour entrainer une interruption de l'écriture. En ce sens, la rature révèle aussi des points de blocage pour le scripteur, l'existence de formes qu'il choisit de rejeter dans le domaine du non (in)scriptible. Un tel choix renvoie à une démarche de régularisation de la langue, et peut manifester, par endroits, l'existence d'une posture ou d'une visée normative. Dans le discours de quelques épistoliers de "Corpus 14 », nous pouvons retrouver, grâce à une recherche de concordance, quelques traces de modalisation axiologique qui renvoient, en creux, à ce que serait leur norme du bien écrire :

(1) « vous me pardonerait sur mon barbouilliage card Je les fait en vitesse je sais pas si vous la pourait lire mon crayon voulez pas marché » (Joseph ou Jules Ramier, 31 août 1917); «Hier je t'ai envoyer une carte de Largentière mais bien mal écrite, tu aura bien dit que j'ai écrit bien de travers, mais à la table que j'étais je n'avais pas beaucoup de place » (Victoria Arcis, 3 octobre 1916)

«mais çi cela ne te dérrenge pas car ce n'est pas un reproche que je te fait; de bien faurmer tes mots car il faut que je praine la suite des frase pour pouvoir lire les lettres et tu n'est pas la ceule car Marie est la méme chose sauf Gabrielle, ou de Louise méme encore il ly ana qu'elque une défoi quil me les faut lire de foi en fin 1 cela ne menpaiche pas de savoir çeque vous vous les dire ; Je pense que çela ne vous fachera pas » (Laurent Pouchet, 10 janvier 1915)

(2) «tu tes mal espliquer sur ta lettre que que tu ma envoyez laurs de ton depart de Baillargues, [...] ai reçu une lettre de Louise en mème temp que la votre date du 5, elle men parle, et me di que jai mal compris, [...] que veutu, ne vous inquiètait pas sur çeula, je prent tout a ma charge, çes moi qui est tout le tord » (Laurent pouchet, 10 septembre 1915); «Tu doi avoir mal conpri sur la lettre çe n'est pas de largent que je te dememde ç'était un petit coli, c'était pour çeula que je 
tedi que çi tu avez qu'èlque chose a menvoyez de te dépècher avan que je parte en permission » (Laurent Pouchet, 19 septembre 1915)

La lettre mal écrite est, pour Joseph, Victoria et Laurent, le plus souvent une lettre difficile à déchiffrer (1), et source d'ambiguïté (2). Les causes sont multiples, certaines explicitées par l'épistolier ou l'épistolière qui s'en excuse auprès du destinataire ; il s'agit dans nos extraits de causes matérielles : un crayon défaillant pour Joseph, une table trop petite pour Victoria (1).

La rature peut elle-même signifier le degré de contrainte ou de liberté du scripteur, « décliner tous les degrés qui conduisent de l'exercice du libre-arbitre à l'aliénation et il est clair que le sens et la portée d'une correction dépendent pour beaucoup de son degré d'indépendance » (Biasi, $2000: 32$ ). Du côté de la contrainte maximale se trouvent les ratures allographes coercitives imposées par la censure ; à l'autre extrémité figurent les ratures autographes libres, les plus communes dans les manuscrits d'écrivains, celles « où le scripteur exerce pleinement et sans contrainte son droit à s'amender et à se contredire, d'autant plus facilement que le manuscrit de travail est, en principe, un document privé [...] où l'écrivain peut "prendre des libertés" avec la plupart des exigences essentielles de l'écriture : syntaxe, orthographe, modèle générique, principe de non contradiction, etc. » (Id., 33). Dans notre corpus, nous l'avons vu (supra 2.3.), toutes les ratures sont autographes et non occultantes, il n'y a pas de ratures apposées par le service de censure. Aucun caviardage donc, mais des phénomènes de silenciation que les poilus signalent au destinataire :

ne sois pas etonee si tu ne reçois pas quelque lettre de celles que je tai dega envoyees il nous est absolument defendu de trop parler (Ernest Viste, 13 novembre 1914)

Je peut pas vous dire ou je suis car c'est defendu, mai je suis pas pret je suis des plus loing (Henri Lorton, 14 décembre 1914)

a partir de maintenant on ne pourras plus dire le pays que l'on ai ce seras interdit (Auguste Foray, 2 janvier 1915)

Je puis pas vous t'espliquer les resultat car il nous est sévérement defendu de donner de renseignement au sujet de la Guerre, car san doute on a vu qu'elque lettre paurtand des choses contre les règiments mes enfin laursque tous sera fini à laur on poura parler à son aise pas en mal mes du bien (Laurent Pouchet, 2 février 1915)

Ces silences, parce qu'ils disent l'existence d'une autocensure, pourraient être considérés comme des formes de ratures autographes blanches. Quand elle n'est pas mentionnée par le scripteur, l'autocensure reste toutefois bien délicate à appréhender pour le linguiste, tout comme le coefficient de liberté ou de contrainte d'une rature, a fortiori quand cette dernière est dite libre, car «c'est précisément dans cet espace de liberté totale que se rencontrent aussi, le plus souvent, les traces d'un système de contrainte intérieur, obscur mais visiblement très actif: lapsus, images obsédantes, retour du refoulé biographique, etc. » (Biasi, 2000 : 33). Et dans le prolongement de ces réflexions, la rature pose aussi la question de l'identité d'une l'instance raturante qui, «par sa dimension d'auto-correction, d'auto-construction réflexive de soi ou d'une image de soi entretient un rapport privilégié avec la question de [...] la représentation de (l'image de) soi » (Rougé, $2000: 10$ ). Cette question de l'éthos sera abordée relativement aux lapsus de personnes que certains de nos épistoliers laissent échapper, et raturent dès que repérés (infra 3.3.). Avant d'évoquer plus largement les ratures libres qui donnent à voir le scripteur en prise avec son activité de réécriture, nous nous attarderons sur un autre type de ratures libres largement représentées dans le corpus, et qui montrent le poids de la norme dans la pratique scripturale des peulettrés au début du XXème siècle, les ratures orthographiques.

\subsection{Rature erratum}


Dans les manuscrits des écrivains, les ratures correctives concernent le domaine de l'orthographe, «celui des éléments lexicaux et/ou syntaxiques » (Biasi, $2000: 32$ ). Les lettres du corpus n'échappent pas à la règle. Sur le corpus dépouillé, la part des ratures dévolues à la correction orthographique n'est pas négligeable, puisqu'elle représente $15 \%$ des reprises effectuées par les scripteurs ${ }^{24}$. Cette attention varie selon les épistoliers : Abel, qui reprend peu ses écrits, n'effectue aucune correction orthographique ; Marie et Louise corrigent peu leur orthographe; pour d'autres, comme Joséphine dont les ratures correctives représentent plus d'un tiers des reprises et Joseph, l'orthographe reste une préoccupation.

Tableau. Proportions des ratures correctives.

Ce tableau présente les proportions des ratures correctives relevées dans les lettres et cartes de neuf scripteurs, dont les écrits totalisent 211653 mots. Les chiffres de la première colonne correspondent aux nombres de corrections, par rature le plus souvent. La deuxième colonne exprime la place de ces ratures correctives relativement à la totalité des mots du scripteur (l'unité de référence est, par commodité logicielle, le mot). La troisième, la part des ratures correctives relativement à la totalité des reprises effectuées par le scripteur. Dans la dernière colonne, le pourcentage renvoie à la place des ratures correctives dans l'ensemble des reprises effectuées par les neuf scripteurs. Joséphine apporte ainsi 12 corrections à ses écrits, soit $0,28 \%$ des mots de son corpus ; 38,7 \% de ses reprises concernent le domaine de l'orthographe, ce qui représente $1,53 \%$ des reprises générales.

\begin{tabular}{|l|c|c|c|c|}
\hline & Corrections & $\begin{array}{l}\text { \% Total mots } \\
\text { scripteur }\end{array}$ & $\begin{array}{l}\text { \% Total reprises } \\
\text { scripteurs }\end{array}$ & $\begin{array}{l}\text { \% Total reprises } \\
\text { corpus }\end{array}$ \\
\hline $\begin{array}{l}\text { Abel } \\
\text { Gombert }\end{array}$ & 0 & 0 & 0 & 0 \\
\hline Alfred Foray & 4 & 0,01 & 16 & 0,51 \\
\hline $\begin{array}{l}\text { Henri } \\
\text { Lorton }\end{array}$ & 9 & 0,12 & 18,36 & 1,14 \\
\hline $\begin{array}{l}\text { Joseph } \\
\text { Ramier }\end{array}$ & 5 & 0,23 & 21,7 & 0,63 \\
\hline $\begin{array}{l}\text { Joséphine } \\
\text { Pouchet }\end{array}$ & 12 & 0,28 & 38,7 & 1,53 \\
\hline Jules Ramier & 34 & 0,05 & 18,27 & 4,33 \\
\hline $\begin{array}{l}\text { Laurent } \\
\text { Pouchet }\end{array}$ & 28 & 0,04 & 13,59 & 3,57 \\
\hline $\begin{array}{l}\text { Louise } \\
\text { Tessier }\end{array}$ & 1 & 0,04 & 8,3 & 0,12 \\
\hline Marie Fabre & 6 & 0,02 & 6,8 & 0,76 \\
\hline Total & 99 & 0,04 & 15,8 & 12,6 \\
\hline
\end{tabular}

Nos scripteurs rectifient certaines de leurs graphies soit en supprimant un élément d'étendue variable (une lettre ou une syllabe à l'intérieur d'un mot; un mot entier; supra 2.3.) par une rature de suppression (1), soit en substituant à la variante initiale un autre élément que le scripteur juge correct $(2)^{25}$. Le substitut est inscrit en surcharge (2a), ou signalé par une rature de substitution (2b). Dans ce cas, le scripteur rature le plus souvent la totalité du segment écrit, qu'il rétablit ensuite dans sa forme selon lui correcte. L'erreur orthographique peut être constatée en cours d'écriture (2c) :

(1) Ils nous parle bien que les boches la pille, mai je crois qu'on va pas tarder non plus (Henri Lorton, 22 avril 1915)

(2a) il commence a pas faire chaud dans les tranchers mai $i \underline{i}<\mathbf{y}>$ faut bien sy habituer (Henri Lorton, non daté); comme désser nous avions deux $b \underline{u}<\mathbf{i}>$ squis chacun (Jules Ramier, 25 décembre 1915) ${ }^{26}$

(2b) ne te fait pas du mauvais sang $\mathrm{q}<\mathrm{c}>$ ar cela $\mathrm{n}$ avance a rien (Jules Ramier, 25 juin 1916); nous ne savons pas ou nous allons c'est probablement dans t'Este $<l^{\prime} E s t>$ (Alfred Foray, 17 août 
1914); pour le bois il la pas encore finis <finit> quand il l'aura finit Jirait (Joseph Ramier, 23 décembre 1915)

(2c) tu me demande surta lettre ce que je praifère un colis ou un $<$ mand $>$ at (Laurent Pouchet, 23 janvier 1915); ne qu'int $<$ ne t'inqu>iète pas chère épouse (Jules Ramier, 17 mars 1915)

Dans l'ensemble, les ratures correctives pointent des erreurs bien connues et répertoriées par les théoriciens et didacticiens de l'orthographe ${ }^{27}$, des erreurs liées aux différents domaines du plurisystème orthographique (Catach, 1989 ${ }^{28}$ ). Les scripteurs rectifient des erreurs de type extragraphique qui affectent la prononciation d'un mot dans lequel il manque un élément (1a), les lettres sont inversées (1b), ou le graphème choisi est erroné (1c) ${ }^{29}$ :

(1a) c'est des petits peignes a barbe en eélıö̈de < céluloïde> (Alfred Foray, 23 novembre 1914) ; l'Eonore $<$ Elèonore (Laurent Pouchet, 16 février 1915); la seinte Virge < vièrge $>$ (Marie Fabre, 21 février 1915)

(1b) elle doit bien plar < parlé> (Jules Ramier, 25 juin 1916); arrivé à notre destati <destination> (Jules Ramier, 26 octobre 1916); tu pourra dire a Abm $<$ Amb $>$ lard (Laurent Pouchet, 2 novembre 1915) ; qu'il puisse un jour nous sarir <secourir $>$ (Marie Fabre, 31 décembre 1915) (1c) J'ai chanch $<\mathbf{g}>$ er de secteur (Henri Lorton, 21 février 1915); il faut at $<$ ou $>$ vrir l'œil et le bon (Laurent Pouchet, 21 novembre 1914)

Les scripteurs repèrent et corrigent aussi des erreurs qui engagent la correspondance phonographique ${ }^{30}$ de la langue écrite : ils modifient les graphèmes erronés, même si leur choix initial n'altère pas la prononciation du mot. Ils rectifient ainsi les digrammes correspondant aux nasales [9] et [*], préfèrent le digramme «au » au graphème simple « $\mathrm{O} »$, hésitent entre deux graphies du « $\mathrm{e}$ » ouvert, et remplacent le graphème « $\mathrm{c}$ » par « $\mathrm{s} »$ pour transcrire le son $[\mathrm{s}]$ :

je vous en $<\mathrm{em}>$ brasse tous du fond du cœur (Laurent Pouchet, 6 décembre 1914)

qui tembrasse pour la vie uns < ainsi $>$ que sa petite fille (Jules Ramier, 11 juin 1916)

c'est acce $<$ au $>$ se de quelques-uns (Pierre Fabre, 12 avril 1915)

a quand pouron nous revoir le beau solai < $<$ soleil $>$ du midi (Laurent Pouchet, $1^{\text {er }}$ octobre 1914);

j'aurais mon bras moins red $<$ raide $>$ (Pierre Fabre, 17 février 1915)

$\underline{C}<\mathbf{s}>i$ tu veu te servir de ces lampes (Jules Ramier, 10 juillet 1916)

Enfin, des ratures correctives dépassent le cadre du graphème, elles rectifient des erreurs de segmentation $^{31}(2)$ et des cas d'homophonies (3) :

(2) si l'on peut entrér a 1 hopital nin $<$ n'im>porte qu'el jour (Marie Fabre, 29 août 1914); la France en a mare et ta L'allemagne de même (Jules Ramier, 5 juin 1916)

(3) Pour vous faire savoir de mes <mais> nouvelle (Joseph Ramier, 8 mai 1918); ont <on> attendant bientot le plaisir de se voir (Joseph Ramier, 14 juillet 1918); je me porte bien, est je souhaite que tu en sois de même (Henri Lorton, 26 février 1915)

D'autres types d'erreurs ne font quant à elles pas ou peu l'objet de ratures. Nous n'avons ainsi relevé dans les lettres étudiées aucune correction en lien avec la ponctuation. Une absence qui pourrait être reliée à la forme même de l'écrit des peu-lettrés ${ }^{32}$ :

«Chez nos épistoliers, [...] les ponctuations doubles [(point d'exclamation, d'interrogation, double point et point-virgule)] représentent $3 \%$ de la ponctuation, la quasi-totalité étant des points d'exclamation. Chez nos neuf épistoliers, l'usage du point et de la virgule diffère de l'usage normé, non seulement par la parcimonie générale dont ils font preuve, mais aussi par leur emploi particulièrement faible de la virgule » (Steuckardt, 2015 : 93-94)

Le domaine de l'orthographe grammaticale fait aussi l'objet d'un traitement particulier de la part de ces scripteurs peu-lettrés. Une étude de leurs usages graphiques (Pellat, 2015 et 2017 ; Surcouf, 2018) a ainsi mis en évidence «le nombre de graphies déviantes par rapport à la norme orthographique, en particulier dans les domaines sensibles et visibles de l'orthographe grammaticale» (Pellat, 2015: 73). Ces variantes graphiques qui se caractérisent notamment par l'absence ou le choix erroné d'un morphème flexionnel «aboutissent à une lecture conforme à la cible» (Surcouf, $2018: 13$ ). Les lettres et cartes que nous avons analysées présentent quelques ${ }^{33}$ ratures en lien avec la correction 
grammaticale, des pratiques inégales selon les scripteurs. Nous trouvons par exemple des corrections d'erreurs relatives à la graphie du mode participial (1), ou de la marque de la personne (2) :

(1) pour le bois il la pas encore <inis <finit> quand il l'aura finit Jirait (Joseph Ramier,23 décembre 1915); le colis que ma soeur Anna ma envoyer le 4 Novembre je ne lai pas vus (Laurent Pouchet, 8 décembre 1914)

(2) vous fetez $<\mathbf{s}>$ pas du mau-vais sang (Henri Lorton, non daté) ; je tes envoyer un colis de linge quand tu laura $<\mathrm{t}>\mathrm{s}$ reçue tu me le diras (Jules Ramier, 14 mars 1918)

Dans des proportions diverses donc, les biffures liées à l'orthographe révèlent la présence d'une pratique corrective. Pour évaluer la proportion réelle qu'occupe ce type de ratures, il conviendrait d'affiner l'analyse en considérant plusieurs paramètres : par exemple le nombre de variantes grammaticales dont la forme normée appellerait une correction par rature $^{34}$; le poids des ratures orthographiques relativement au domaine de l'orthographe ; les usages des scripteurs qui aideraient à définir des zones de stabilité et d'instabilité corrective $^{35}$ dans leur pratique scripturale. La présence de ces ratures révèle le degré d'attention, de vigilance (voire de tolérance pour les formes non corrigées) que porte le scripteur peu-lettré à la question de la norme graphique, et, par capillarité peut-être aussi le poids dans l'enseignement du début du XIX siècle de l'orthographe (voir notamment Chervel, 1991). Plus, il est des ratures dont l'absence même peut être particulièrement signifiante, quand elle est interprétée à l'aune des routines graphiques des scripteurs peulettrés ${ }^{36}$. Joseph, qui confond par exemple parfois les nasales [9] et $[7]^{37}$, ne rectifie qu'une de ses variantes dont il ne change pas pour autant le son non normé :

«Bien cher frère Je vais te terminer pour aujourd hui ent $<$ on $>$ attendant bientot le plaisir de se voir » (Joseph Ramier, 14 juillet 1918).

Ce type de rature, et l'absence des autres, nous laissent à l'intersection de deux interprétations opposées : soit elle est le signe d'une mauvaise discrimination phonologique propre aux scripteurs dont le processus d'acquisition orthographique n'a pas été achevé, soit elle est la trace d'une variation diatopique ${ }^{38}$, voire d'un idiolecte.

\subsection{Ratures et r(é)écritures}

Une autre catégorie de ratures de notre corpus donne à lire un scripteur en prise avec sa pratique d'écriture, au sens large du terme. Nous proposons ici, à travers quelques exemples choisis de ratures libres, de suivre « les traces d'une écriture en acte » (Fenoglio, $2003: 57)$.

Un premier type de rature permet au scripteur d'ajouter au fil de son écriture une précision de dernière minute ${ }^{39}$. Cette démarche se manifeste par la suppression temporaire d'un élément que le scripteur rétablira à l'identique dans le cotexte droit de sa phrase, un empan non nul séparant le terme biffé de son doublon. Ce type de rature sur le fil est particulièrement représenté dans les lettres d'une de nos scriptrices, Marie Fabre $^{40}$ :

Ils nous on dit que le landemain il serait au soulie mais alors il a eu changé d'idée il ma dit \{le dimanche matin $\}<$ il ma dit> que il fallait qu'il aille vendangér et qu'il ne pouvait pas venir $(9$ octobre 1914)

a Grand Dieu aumerons a ton arrivé $<$ nous aimerons $>$ bien mieu que se soit fait que non pas a faire (31 décembre 1915)

tu etait bléssé jai dit dessuitte que ou que tu sois je voulez venir te voir même je croyai bien le faire aid a car personne ne me disait non $<$ mais apresant $>$ que le moment est arriver personne ne le veut (29 août 1914)

il faut espérer que le bon Dieu te le tiendra en compte et $\{$ car tu as fait ton devoir\} <et> croi cher Pierre que pour moi ainsi que tout nos parents nous ne sommes pas des plus mal heureux ( 3 octobre 1914) 
sils ne tavait pas gardé pauvre \{bléssé come tu etai\} < tu $>$ naurais pas eu la force de faire tout le chemin que tu as fait (3 septembre 1914)

je ne pourrai jamais savoir coment tu étais a ton arrive du champ de bataille mais \{c'est un peu pénible pour tous deux chéri\} <mais> nous vivons avec se grand éspoir de nous revoir (20 septembre 1914)

Marie revient sur son écrit pour situer le cadre temporel dans lequel s'inscrit un discours rapporté ou un événement espéré, pour expliquer son propos ou pour signifier pudiquement un sentiment de lassitude. Ces repentirs ajoutés in extremis donnent à lire une phrase qui progresse par allongements. Ils façonnent le contour rythmique de la phrase de Marie qui se caractérise par son phrasé si particulier, sa «scansion régulière » (Steuckardt, 2015: $100)^{41}$.

D'autres ratures dans le corpus présentent en partie les mêmes caractéristiques que ces ratures de précision, à ceci près qu'elles rectifient un dysfonctionnement de la chaine écrite: le scripteur rature un segment intrus qui, survenu trop tôt, vient enrayer la mécanique syntaxique de la phrase, puis il le reproduit ${ }^{42}$ à sa juste place dans la suite de son discours :

Je te qu dit <qu>e un un ces jours je te R'onvoy erai de l'argent (Jules Ramier, 17 octobre $1914)^{43}$

La derniere dan fois $<$ dans $>$ une lettre (Jules Ramier, 23 novembre 1917)

Je net pas bien le temp il faut aller chercher et la soupe <et $>$ les lettres (Jules Ramier, 11 juin 1916)

Dit moi le sur $<\mathrm{ta}>$ letre (Marie Fabre, 3 février 1915)

Ce type de ratures anticipatoires (Anokhina, $2018: 131)$ résulte d'une « désynchronisation des processus cognitifs » (Ibid.) engagés dans le processus rédactionnel : l'attention du scripteur est portée sur la planification de son discours, l'échappé raturé est un mot provenant du dire à venir. Ce type d'anticipation est due au traitement parallèle des opérations cognitives impliquées par l'acte d'écriture, et est peut-être causé par les conditions dans lesquelles les scripteurs produisent leurs écrits, contraints par un petit moment ou instant de libre (supra 2.1.) :

«Pour maximiser la vitesse de production, et si [...] la capacité le permet, les processus peuvent s'engager parallèlement. Dans ce cas, l'attente par un processus du produit de l'autre suppose que ces processus traitent parallèlement des unités différentes. Ainsi, le processus de planification élabore une portion du contenu sémantique qui est transmise au processus de formulation. Pendant que celui-ci encode ce contenu, le processus de planification peut d'ores et déjà traiter en parallèle la portion suivante du contenu sémantique. Selon ce principe, une unité donnée est traitée séquentiellement, mais les trois processus traitent simultanément trois unités différentes » (Plane, Alamargot, Lebrave, $2010: 17$ )

Ces ratures anticipatoires trahissent donc une forme d'aliénation cognitive du scripteur pris dans l'élaboration de son dire à venir.

Quand le déraillement de l'écrit est le produit d'un acte manqué, d'un raté, il nous conduit sur le chemin, plus épineux, des ratures de lapsus. Les lapsus ont la même valeur indicielle que le signe pictural pour Morelli : «elles témoign[ent] des moments où la vigilance de l'artiste, lié par la tradition culturelle, se relâch[e] pour laisser place à des traits purement personnels "qui lui échapp[ent] sans qu'il en [ait] conscience" » (Ginzburg, 1980 : 8). Dans notre corpus, certains mots raturés peuvent être interprétés comme des lapsus repérés par les scripteurs. Ce type de rature, qui atteste l'existence d'un lapsus, « opère un décrochage sémiotique entre deux trajets énonciatifs en principe séparés et qui là se rencontrent dans l'événement-lapsus; la rature rectifie et tente d'occulter l'événement dans l'après-coup mais elle montre, le plus souvent, le signifiant raturé [...]» (Fenoglio, 2003: 63). Les épistoliers rayent essentiellement des lapsus de $\operatorname{mot}^{44}$, des lexies identifiables qui dévient de l'intention initiale du scripteur ${ }^{45}$, et leur substituent immédiatement le mot cible, qui apparait en position intralinéaire ${ }^{46}$ :

(1) les journées son trais $<$ chaudes $>$ (Laurent Pouchet, $1^{\text {er }}$ octobre 1914); 
(2) je te dirais qu'on se trouve dans un patelain ou il y a $<$ beaucoup $>$ de vigne et du bon vin (Henri Lorton, 16 février 1915)

(3) Je ne set pas bien si il $\mathrm{n}$ y en a pas de <blessés> (Jules Ramier, 6 juillet 1916)

$\mathrm{Au}$ sein des paradigmes adjectivaux ou adverbiaux, les termes sont associés par une relation antonymique ${ }^{47}$ ou une euphémisation. Nous souhaitons porter notre attention ici sur les ratures des lapsus qui renvoient à des paramètres énonciatifs du genre épistolaire, et plus particulièrement celles qui concernent les références aux personnes engagées dans l'énonciation de discours. Rappelons que les épistoliers écrivent plusieurs lettres par jour, à des destinataires différents, parfois regroupés sur une même lettre. Rappelons aussi que l'un d'eux, Jules Ramier, n'écrit pas ses lettres lui-même. Les lettres portent les traces de ces conditions de production, les ratures de lapsus révélant une certaine confusion de la part des épistoliers qui signent :

ta fenme <sœur> pour la vie (Joséphine Pouchet, 20 mars 1916)

ton époux devoue Louis Hilaire <Jules Ramier> (Jules Ramier, 13 mai 1917)

Joséphine écrit à son frère et non à son époux, et le second lapsus nous apprend l'identité du soldat qui a écrit tout ou partie des lettres de Jules Ramier. Une même lettre peut être destinée aux parents, à l'épouse et éventuellement aux enfants, auxquels le soldat s'adresse successivement ou collectivement. Et les lapsus affectant les déictiques désignant les destinataires montrent combien il peut être compliqué pour certains, comme Laurent Pouchet ici, de passer du « tu » individuel au « vous » collectif, et inversement :

Je puis pas $<t^{\prime}>$ espliquer les resultat car il nous est sévérement defendu de donner de renseignement au sujet de la Guerre (2 février 1915)

<teu> men vaira qu'el-ques mouchoirs, car il men réste que deux ( $1^{\mathrm{er}}$ mars 1915$)$

Je fais réponse a ton aimable lettre la qu'elle ma fait plaisir sur tout en na prenand que $<$ tu $>$ es toujour en bonne et parfaite santé (28 juin 1915)

Tu me di que ma tante ta <vous> a ècri (16 mai 1915)

ébien $<$ vous $>$ pouvez croire que je lai mettré dans une lettre ( $1^{\text {er }}$ juillet 1915 )

Les ratures de lapsus concernent aussi la première personne ${ }^{48}$, le «je» écrivant qui se distingue difficilement du destinataire individuel, désigné par «tu» (1) ou qui se fond d'abord dans un «nous » ou un « on » collectif (2) :

(1) quand tu m'écrira tu me dira si < <on> frère joseph et parti (Jules Ramier, 4 avril 1916)

je pense qu'il la n'est de méme pour moi < vous $>$ autre (Laurent Pouchet, 6 décembre 1914)

Je te dirrai que jai reçu limpèrmeable hier 29 , il est arriver en bon état, <je $>$ suis bien comptemt, mais il est trop joli pour mettre dans les tranchées (Laurent Pouchet, 30 décembre 1915)

(2) Je vien par cette lettre vous faire savoir létat de $<$ ma $>$ santé (Laurent Pouchet, 23 juin 1915)

maintenant en $<$ je $>$ me trouve dans un beau pays de vin (Henri Lorton, 23 février 1915)

jai recut ton manda et je te remercie car cela nous $<$ me $>$ permettra de boire quelque litres de pinar (Jules Ramier, 30 mars 1916)

je ne peu pas te Dire si j'irai en permisson mais j'ai bien peur que nous $<\mathbf{J e}>$ ne l'ai pas (Jules Ramier, 8 août 1918)

Dans ces dernières occurrences (2), le scripteur, dans un premier mouvement d'écriture, se désigne au moyen d'un nous exclusif ${ }^{49}$ ou groupal, par lequel il signale son appartenance à la communauté des soldats :

Les dangers constants de mort et la vie souterraine dans la boue des tranchées ont favorisé non seulement la nécessité, de la part des soldats, d'entretenir des liens avec l'arrière, avec la vie civile, mais aussi la création d'une identité groupale, d'un nous par lequel tout poilu se fait le porte-parole de l'ensemble des combattants (Vicari, 2012:682)

Ce type de lapsus témoigne de l'importance, ou pour le moins atteste l'existence d'un éthos collectif porté par le pronom nous, plus rarement on, et auquel le scripteur recourt en premier pour se désigner au destinataire. L'apparition du nous collectif à la suite d'un « je » individue $l^{50}$ peut être motivée par le contenu du propos qui ramène le « je » épistolier à son 
quotidien de guerre, à ce qui fait le groupe auquel il appartient, comme une expérience ou des préoccupations partagées par les soldats : l'évocation d'une permission attendue certainement par bon nombre de soldats, l'endroit de cantonnement de la compagnie, les vertus lénifiantes du vin, et, bien entendu, la santé ${ }^{51}$. Dans ces exemples, le scripteur choisit de raturer les pronoms qui réfèrent au groupe, et qui n'entament pour la plupart pas la lisibilité de son propos; et peut-être la lettre est-elle cet unique endroit où le "je» épistolier, s'adressant à sa famille restée à l'arrière, peut se détacher sans risque du groupe ? Quoi qu'il en soit, ces lapsus de personnes grammaticales qui engagent la construction d'un éthos "poilu», comme les ratures de lapsus qui, dans les formules liminaires des lettres, concernent les références spatiales ou temporelles, représentent selon nous des lapsus-discours, ces "lapsus récurrents dont la récurrence fait discours" (Fenoglio, ITEM) ; ils aident à saisir la complexité d'une énonciation épistolaire en temps de guerre.

\section{Conclusion}

Les ratures qui, par endroits, émaillent nos correspondances peu-lettrées de la Grande Guerre sont certes moins nombreuses que celles que l'on pourrait trouver sur un brouillon, et ne retracent pas les métamorphoses successives d'un même écrit comme le feraient les ratures d'écrivains. Ces ratures de peu-lettrés n'en forment pas moins un matériau riche et complexe : elles trahissent un geste scriptural de l'instant qui parfois dysfonctionne, elles donnent à voir un scripteur peu-lettré attentif à sa graphie, elles tracent les mouvements d'une écriture emportée par les rails de la pensée, et qui trébuche par endroits. Et, quand elles s'enchainent, les bifurcations nous laissent à la croisée des chemins, comme dans cette phrase de Marie Fabre à qui nous laissons le dernier mot :

«Enfin mon chèr Pièrre c'est triste pour nous e'est mais moi croi le de ne pas pouvér te soignér mais vois tu depuis ton départ je ne fesai que prier Dieu qu'il est la bonter de técarter du dangér de ces vilain d'allemants » (29 août 1914)

\section{Références bibliographiques}

ANOKHINA, O. (2018). Les corrections anticipatoires. Résultat de la désynchronisation des processus cognitifs lors de l'écriture, in O. Anokhina, et al., La fabrique du texte à l'épreuve de la génétique, [Brest], Archives contemporaines, 129-154.

BiASI de, P.-M. (2000). Qu'est-ce qu'une rature?, in B. Rougé (éd.), Ratures et repentirs. Pau : Presses Universitaires de Pau et des Pays de l'Adour, 17-47.

BRAncA-Rosoff, S. (2017). La Grande Guerre des ruraux peu-lettrés : une expérience populaire d'écriture, in O. Roynette, G. Siouffi et A. Steuckardt (éd.), La langue sous le feu. Mots, textes, discours de la Grande Guerre. Rennes : Presses Universitaires de Rennes, 111-123.

CATACH, N. (1989). L'orthographe française. Paris : Nathan.

Chervel, A. (1991). L'école républicaine et la réforme de l'orthographe (1879-1891). Mots Orthographe et société, 28, 35-55.

FABRE, D. (1993). Écritures ordinaires. Paris : P.O.L.

Fenoglio, I. (2003). Graphie manquée, lapsus écrit: un acte d'énonciation attesté, Langage et société, 103, 55-77.

Fenoglio, I. (2006). Les événements d'énonciation graphique. Traces du fonctionnement linguistique de l'inconscient dans les manuscrits, ITEM.

http://www.item.ens.fr/articles-en-ligne/les-evenements-denonciation-graphique/, consulté le 28 août 2019. 
FonviElle, S. (2020 à par.). Lettres de peu-lettrés durant la Grande Guerre. Une écriture sur le fil de la rature, in A. Steuckardt, C. Gomila, Ch. Wionet (éd.), Gens ordinaires dans la Grande Guerre : correspondances, récits, témoignages, Editions de la Maison des Sciences de l'homme.

GEA, J.-M. (2015). Le dialecte dans l'écriture de la guerre, in A. Steuckardt (éd.), Entre village et tranchées. L'écriture de Poilus ordinaires. Uzès : Inclinaison, 157-167.

GinzBurG, C. (1980). Signes, traces, pistes. Racines d'un paradigme de l'indice, Le Débat, 6, 3-44.

Gresillon, A. (2000). Raturer, rater, rayer, éradiquer, irradier, in B. Rougé (éd.), Ratures et repentirs. Pau : Presses Universitaires de Pau et des Pays de l'Adour, 49-60.

Gresillon, A., LeBrave, J.-L. (2003 [1982]). Les manuscrits comme lieu de conflits discursifs. in C. Fuchs et al. (éd.), La Genèse du texte : les modèles linguistiques. Paris : Éditions du CNRS, 129175.

Große, S., Steuckardt, A., Dal Bo, B., Sowada, L. (2016). Du rituel à l'individuel dans des correspondances peu lettrées de la Grande Guerre. Actes du 5e Congrès Mondial de Linguistique Française.

KIVINIEMI, A.-L. (2019/3). Identité verbale et modèles d'expression dans les lettres de poilus, in Mots, les langages du politique, 121, 109-130.

Lucci, V., Millet, A. (1994). L'orthographe de tous les jours. Enquête sur les pratiques orthographiques des Français. Paris : Champion, coll. « Politique linguistique ».

LuXARDO, G. (2015). Fréquences des colis et marmites : comment mesurer la languitude ? in A. Steuckardt (éd.), Entre village et tranchées. L'écriture de Poilus ordinaires. Uzès: Inclinaison, 113-123.

Plane, S., Alamargot, D., Lebrave, J.-L. (2010). Temporalité de l'écriture et rôle du texte produit dans l'activité rédactionnelle, Langages, vol. 177, 1, 7-28.

Pellat, J.-Ch. (2015). Les graphies des Poilus. Loin des canons orthographiques, in A. Steuckardt (éd.), Entre village et tranchées. L'écriture de Poilus ordinaires. Uzès : Inclinaison, 67-77.

Pellat, J.-Ch. (2017). Les difficultés de mise à l'écrit des peu-lettrés : les graphies des Poilus, in Kristol, A. M. (éd.), La mise à l'écrit et ses conséquences. Tübingen: A. Francke, 237-245.

Rey-Debove, J. (2003 [1982]). Pour une lecture de la rature, in C. Fuchs et al. (éd.), La Genèse du texte : les modèles linguistiques. Paris : Éditions du CNRS, 21-72.

Rouge, B. (2000). La rature, le repentir et "l'avoir-eu-lieu”, in B. Rougé (éd.), Ratures et repentirs. Pau : Presses Universitaires de Pau et des Pays de l'Adour, 7-14.

Rossi, M., Peter-Defare, E. (1998). Les lapsus. Ou comment notre fourche a langué. Paris : Presses Universitaires de France.

Steuckardt, A. (2014). De l'écrit vers la parole. Enquête sur les correspondances peu lettrées de la Grande Guerre, Actes du 4e Congrès Mondial de Linguistique Française.

Steuckardt, A. (éd.) (2015). Entre village et tranchées. L'écriture de Poilus ordinaires. Uzès: Inclinaison.

SteuckardT, A. (2015). Sans point ni virgule, in A. Steuckardt (éd.), Entre village et tranchées. L'écriture de Poilus ordinaires. Uzès : Inclinaison, 91-100.

Steuckardt, A., GeA, J.-M., Fonvielle, S. (2017). La rencontre de l'écrit. Langue nationale et substrat dialectal dans les correspondances peu-lettrées de la Grande Guerre, in RoYNETTE, Odile; Siouffi, Gilles ; Steuckardt, Agnès (éd.). Rennes : Presses Universitaires de Rennes, 99-110.

Surcouf, Ch. (2018). "tu me di que je ne connai pas lecri ture ébien çi chaque foi que tu écri je le connai" - Analyse des graphies des formes verbales dans la correspondance des Poilus du Corpus14, Actes du $3 e$ Congrès Mondial de Linguistique Française.

VICARI, S. (2012). L'éthos "poilu” dans les lettres des soldats de la Grande Guerre : hétérogénéités énonciatives et stratégies discursives, Actes du 3 e Congrès Mondial de Linguistique Française.

\section{Sitographie des ressources consultées}

Corpus 14 : https://www.univ-montp3.fr/corpus14/index.html

Portail de textométrie TXM de Praxiling : http://textometrie.univ-montp3.fr/txm/

Ortolang : https://www.ortolang.fr/market/corpora/corpus14/7 
${ }^{1}$ Ces écrits privés ont été collectés dans le cadre du projet «Corpus 14 » initié en 2014, sous la direction d'Agnès Steuckardt. Ils sont désormais numérisés, en accès libre sur ortolang ou consultables via la plateforme lexicométrique TXM.

${ }^{2}$ Version la plus récente, « corpus $1420 »$ comprend 469464 mots et ponctuation.

${ }^{3}$ Abel Gombert, Alfred Foray, Alphonse, André Tétart, Auguste Foray, Emile Foray, Ernest Viste, Henri Lorton, Joseph Ramier, Joséphine Pouchet, Jules Ramier, Julie Ramier, Laurent Pouchet, Léonie Ramier, Louis Thomasse, Louise Tessier, Marie Fabre, Pierre Fabre, Régis Landy, Victoria Arcis, Victorin Folier, Claudia Tarest, François Guillaudeau, Jean-Marie Ramier, Joseph Moumier et Sœurs de Saint Joseph.

${ }^{4}$ "D'un point de vue "conceptionnel", la correspondance familiale des Poilus, qui se situe plus près d'une conception parlée que d'une conception écrite de la communication, relève [...] du "parlé graphique" ». (Steuckardt, $2014: 354$ ).

5 Elles «s'opposent nettement à l'univers prestigieux des écrits que distinguent la volonté de faire œuvre, la signature authentifiante de l'auteur, la consécration de l'imprimé. Et puis, surtout, la plupart de ces écritures-là, associées à des moments collectifs ou personnels intenses ou bien à la routine des occupations quotidiennes, semblent vouées à une unique fonction qui les absorbe et les uniformise : laisser trace. » (Fabre, $1993: 11$ )

${ }^{6}$ On retrouve dans les lettres de différents scripteurs (Alfred Foray, 23 mars 1915 ; Ernest Viste, 6 novembre 1914; Marie Fabre, 28 novembre 1914) les formules figées «je profite d'un petit moment».

${ }^{7}$ «C'est à ses ratures que l'on reconnait, au premier coup d'œil, un brouillon, un manuscrit de travail. » (Biasi, $2000: 19)$

${ }^{8}$ Seul Alfred Foray mentionne l'usage d'un brouillon, mais dans le cadre d'une correspondance administrative : «j'en est profiter pour écrire a tous ceux qui n'avait pas payer une carte militaire sous enveloppe. je te mais le brouillon dans ma lettre j'ai dit a tous a peut pret la même chose » (11 février 1915).

${ }^{9}$ Pour mesurer le degré de ritualisation de certaines expressions, ou des formules liminaires des lettres par exemple (Branca-Rosoff, 2015).

${ }^{10}$ Voir notamment Luxardo, 2015.

${ }^{11}$ Nom inconnu.

${ }^{12}$ Pour une ventilation concernant les 26 épistoliers, et le détail des résultats obtenus, voir Fonvielle, à par.

${ }^{13}$ Identifiés dans TXM comme : Claudia Tarest, François Guillaudeau, Jean-Marie Ramier, Joseph Moumier et Sœurs de Saint Joseph.

14 «[...] Il y a d'une part, synonymie entre raturer, rayer et radier, qui signifient tous trois « annuler». Il y a, d'autre part, le double sens de radier, qui signifie à la fois « annuler» et «briller d'un vif éclat ». N'est-ce pas alors la langue elle-même qui suggère le paradoxe selon lequel la rature sert à la fois à « annuler » et à « irradier »? » (Grésillon, $2000: 54$ )

${ }^{15}$ Nous n'aborderons que les propriétés les plus signifiantes pour le corpus. Pour une liste détaillée des critères, voir la grille typologique proposée par Pierre-Marc de Biasi (2000).

${ }^{16}$ Précisons que Jules Ramier fait écrire ses lettres par quelqu'un d'autre, dont l'identité est possiblement révélée par un lapsus de signature (infra 3.3.).

${ }^{17}$ Dans «Corpus 14 », pas de cartes préremplies qui impliquaient une rature sur un tapuscrit allographe.

${ }^{18}$ Le substitut est placé entre crochets $<>$ dans les exemples.

${ }^{19}$ Le scripteur substitue, au sein du paradigme grammatical, deux prépositions.

${ }^{20}$ En italiques le doublon resitué.

${ }^{21}$ Les ratures de syntagmes sont assez rares : «papa m'enverra des sous une dizaine de francs du plus vite possible (Henri Lorton, non daté) »

${ }^{22}$ Signalé par $/ /$.

${ }^{23}$ Les surcharges, qui peuvent être ajoutées immédiatement ou postérieurement, ne peuvent être datées.

${ }^{24}$ Nous avons laissé de côté les corrections dont la visée orthographique n'était pas évidente : correction de la forme graphique ou changement de temps (j'ai écrit) dans «Jai- J'écri a 
Elèonore » (Laurent Pouchet, 3 mars 1915) ; correction et influence du support (fin de ligne) pour « sur tout en na prenan // prennand (Laurent Pouchet, 26 mars 1915).

${ }^{25} \mathrm{La}$ forme corrigée ne correspond pas toujours à la forme normée : six <çi> tu voyer ça (Laurent Pouchet, 24 janvier 1915)

${ }^{26}$ L'élément de substitution en surcharge est encadré par $<>$ et mis en gras, l'élément d'origine apparait en caractères italiques et souligné.

${ }^{27}$ Ajoutons que les ratures correctives portent particulièrement sur les noms propres, toponymes (corpigny <coupigny $>$; Sains-en Chorlle $<$ Choelle $>$ ) ou gentilés (entoines Entoine; Abm $<$ Amblard $>$; Eonore <Eléonore $>$ ). L'onomastique représente un domaine couramment associé à la rature (Biasi, $2000: 32$ ).

${ }^{28}$ Voir la grille typologique des erreurs d'orthographe de Nina Catach (1989) dont nous utilisons la terminologie.

${ }^{29}$ Il s'agit d'erreurs à dominante phonétique dans la typologie de Catach.

${ }^{30}$ Erreurs de type phonogrammique, qui n'altèrent le plus souvent pas la prononciation du terme.

${ }^{31}$ Maladroitement parfois : demain je ten ferai une plus plulonge (Jules). Précisons que les ratures de segmentation ne sont pas nombreuses, alors que la sur- ou sous-segmentation fait partie des usages graphiques de nos scripteurs peu-lettrés, notamment dans l'entourage du verbe (Surcouf, 2018), et " on pourrait se demander si les graphies de type "jelevoi souvant" (LP- 1914.10.28) ne seraient pas le reflet d'une intuition langagière sur le fonctionnement des clitiques, dans la lignée des suggestions de Creissels [...] » (Surcouf, $2018: 11$ ).

32 Reflet peut-être du traitement périphérique que le système scolaire de l'époque réservait à l'enseignement de la ponctuation (Pellat, 2015).

${ }^{33}$ Pour ne pas dire peu.

${ }^{34}$. Certaines erreurs grammaticales pourraient appeler des ratures plutôt suppressives (par exemple la guerre seras) ou plutôt substitutives (par exemple tu $m e$ dit $\langle s>$ ), avec une variable d'ajustement toutefois (substitution au lieu de suppression dans as $<\mathrm{a}>$ ) : " Tu me dit sur ta lettre que le gouvernement vous as adit que la guerre seras finie a la fin Juillet» (Jules Ramier, 18 juin 1915). D'autres corrections, parce qu'elles relèveraient de l'ajout, pourraient se trouver hors du périmètre de la rature (sauf à considérer ici aussi la rature du mot, puis sa réécriture avec ajout) : « Dimanche nous avon eut une carte » (Louise Tessier, 16 septembre 1914); «Bien des carresse au petit ange adoré Albertout» (Laurent Pouchet, 20 février 1915); «la bonne résolution que tu ma donné » (Marie Fabre, 29 août 1914)

${ }^{35}$ Sur le modèle des zones de fragilité ou d'instabilité du système orthographique français (Lucci et Millet, 1994).

${ }^{36}$. Pour une description des routines graphiques de certains épistoliers du corpus, voir Pellat, 2015 : 74-75.

37 « Il a dit de t'on voyé bien le bonjour », « il ton voie un grand bonjour » (23 décembre 1915); « en vous embrasson » (28 novembre 1917, 8 mai 1918), « en ton brasson» (23 décembre 1915); «Je termine on te souhaiton aussi h une bonne heureuse année » ( $1^{\mathrm{er}}$ janvier 1918). Joseph Ramier est né en Ardèche.

${ }^{38}$ Voir au sujet de la prononciation dans les lettres de poilus peu-lettrés : Steuckardt, 2014 ; Géa, 2015 ; Steuckardt, Géa, Fonvielle, 2017

${ }^{39}$ Dans les occurrences citées, les accolades encadrent l'élément inséré.

${ }^{40}$ Nous retrouvons, dans des proportions moindres cependant, ces ratures de précision dans d'autres lettres du corpus : " voila plusieur fois que ca arrive je \{un jour\} <je $>$ ne recois rien et le jour suivent j'en recois deux » (Alfred Foray, 30 décembre 1914); «la-bas la guerre est finie est ici \{c'est pas pareil çà tapes toujours et\}<nous> savons pas quand elle finira » (Jules Ramier, 25 novembre 1918); « il pleu mai $\Theta$ \{malgré çela rien nous arraite $<0>$ n marche » (Laurent Pouchet, 4 janvier 1915); c'est $\mathrm{M}\{$ le pauvre $<\mathrm{M}>$ ournet (Laurent Pouchet, 16 février 1915)

${ }^{41}$ Pour une analyse du phrasé de Marie, voir Steuckardt, 2015.

${ }^{42}$ Le doublon est placé entre crochets.

${ }^{43}$ Il n'est pas rare que «l'instance de contrôle censure une anticipation dès la première syllabe » (Anokhina, $2018:$ 140)

${ }^{44}$ Qui peuvent être distingués des lapsus de syllabes, de voyelles et de consonnes (Rossi et PeterDefare, $1998: 37$ ) 
45 « [U]ne cible est remplacée par un intrus de même classe linguistique, susceptible par conséquent de postuler la position usurpée (Garrett, 1980) » (Rossi et Peter-Defare, 1998 : 35)

${ }^{46}$ Pour une analyse de différents lapsus de mots dans les lettres des peu-lettrés, et la difficulté de les circonscrire, voir Fonvielle, à par.

${ }^{47}$ Sans surprise, car «[...] les études en psychologie cognitive ont montré que la probabilité d'association entre deux antonymes est la plus forte par rapport à l'association avec des termes sans aucun lien sémantique » (Anokhina, $2018: 141$ ).

${ }^{48}$ Une étude de l'usage des pronoms personnels dans un corpus restreint d'écrits de lettrés et de peulettrés a mis en évidence qu' " en ce qui concerne l'identité individuelle, le recours à la personne subjective recule avec le degré d'instruction du scripteur » (Kiviniemi, 2019: 118), les peu-lettrés ayant « une approche plus dialogale de la communication épistolaire, se conformant ainsi aux instructions des manuels primaires : «La lettre est une conversation écrite entre personnes qui ne peuvent facilement se voir a cause de l'éloignement » (Viala, 1896, p. 257) » (Ibid.)

${ }^{49}$ Le nous exclusif «Je + les soldats » est distingué du nous inclusif « Je + destinataire(s)» et du nous inclusif large « Je + communauté non limitée » (Kiviniemi, $2019: 115$ )

${ }^{50}$ Sauf pour la deuxième occurrence, précédée d'un discours dominé par le « on » collectif: « Je viens de Belgique, et c'etait presque le moment, car je comence a on avoir marre, depuis le temps qu'ont y était; et les bains quont y prenaient, car ont $¥$ voyaient que de l'eau » (Henri Lorton, 23 février 1915)

${ }^{51}$ Qui arrive en tête des préoccupations des soldats dans leurs lettres (Luxardo, 2015). 